\title{
Two-dimensional Speckle Tracking Imaging as a Novel Strategy for Accurately Identifying the Accessory Pathway
}

\author{
Tadateru Iwayama ${ }^{1}$, Takanori Arimoto ${ }^{1}$, Harutoshi Tamura ${ }^{1}$, Masahiro Wanezaki ${ }^{1}$, \\ Shintaro Sasaki ${ }^{1}$, Satoshi Nishiyama ${ }^{1}$, Daisuke Kutsuzawa ${ }^{1}$, Daisuke Ishigaki ${ }^{1}$, \\ Joji Nitobe $^{1}$, Hiroshi Suzuki ${ }^{2}$, Tetsu Watanabe ${ }^{1}$ and Isao Kubota ${ }^{1}$
}

\begin{abstract}
A 14-year-old patient was diagnosed with hypertrophic cardiomyopathy associated with Wolff-ParkinsonWhite syndrome. The two-dimensional speckle tracking strain method showed normal left ventricular local contraction, but the peak systolic longitudinal strain of the right ventricular (RV) anterior wall was earlier than that of the septal wall. As expected, the location of the accessory pathway was at the RV anterior wall. The patient's RV local contraction was normalized by successful radiofrequency application.
\end{abstract}

Key words: Wolff-Parkinson-White syndrome, two-dimensional speckle tracking imaging, catheter ablation

(Intern Med 53: 1527-1530, 2014)

(DOI: 10.2169/internalmedicine.53.2019)

\section{Case Report}

A 14-year-old boy was referred to our hospital for the management of a series of sudden-onset palpitations associated with supraventricular tachycardia. At nine years of age, he was diagnosed with hypertrophic cardiomyopathy associated with Wolff-Parkinson-White (WPW) syndrome. A genetic analysis was not performed because his parents did not provide their consent. The plasma levels of B-type natriuretic peptide (BNP) were $275.7 \mathrm{pg} / \mathrm{mL}$, and a chest X-ray showed mild cardiomegaly (cardiothoracic ratio of $49.1 \%$ ). An electrocardiogram (ECG) revealed positive delta waves at I, II, III, aVF, aVL, and V1-6, as well as extremely high $\mathrm{R}$ waves in the left precordial leads. This was suggestive of left ventricular (LV) hypertrophy, despite the presence of WPW syndrome (Fig. 1A). Prior to the electrophysiological study, we performed an echocardiographic examination (Vivid E9 ultrasound system, GE Healthcare, Wauwatosa, USA). We observed concentric LV hypertrophy (19 mm) with a normal end-diastolic dimension of $40.6 \mathrm{~mm}$ and normal LV wall motion with fractional shortening of $45.1 \%$. Two-dimensional speckle tracking (EchoPAC PC software, version 7.0.0, GE Healthcare) did not show any evidence of LV regional deformation. Notably, the peak systolic longitudinal strain of right ventricular (RV) anterior wall was earlier than that of the septal wall (Fig. 1B). It took 15 minutes to perform two-dimensional speckle tracking and a subsequent analysis. Based on the echocardiographic findings, the location of the accessory pathway was speculated to be the $\mathrm{RV}$ anterior wall.

An electrophysiological study and catheter ablation were performed after obtaining written informed consent. As expected, the earliest ventricular activation site existed at the RV anterior wall during sinus rhythm. The earliest atrial activation site during RV stimulation was confirmed to be the anterior side of the tricuspid valve annulus. We successfully applied radiofrequency to eliminate the patient's accessory pathway conduction (Fig. 2). The fluoroscopy and procedure times were 12 and 48 minutes, respectively. The radiofrequency catheter ablation procedure narrowed the QRS interval (Fig. 3A), and two-dimensional speckle tracking imaging showed normalization of the RV regional deformation (Fig. 3B). The patient has subsequently been well and has not required any medication. His plasma levels of BNP decreased to $103.4 \mathrm{pg} / \mathrm{mL}$, and no recurrence of tachycardia

${ }^{1}$ Department of Cardiology, Pulmonology, and Nephrology, Yamagata University School of Medicine, Japan and ${ }^{2}$ Department of of Pediatrics, Yamagata University School of Medicine, Japan

Received for publication October 29, 2013; Accepted for publication February 16, 2014

Correspondence to Dr. Takanori Arimoto, t-arimoto@med.id.yamagata-u.ac.jp 
A

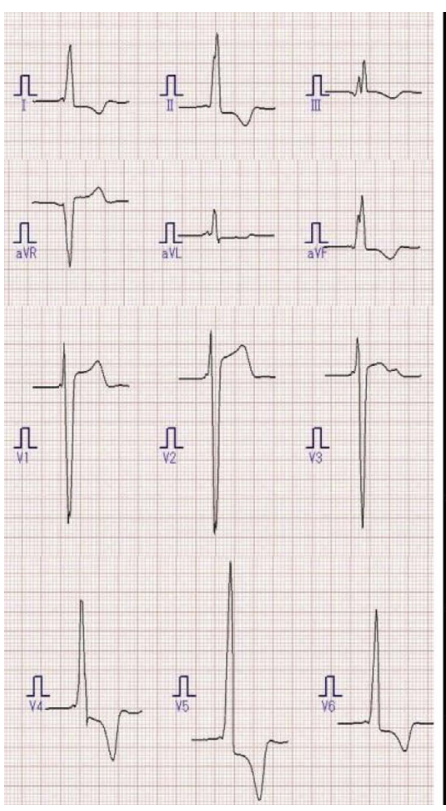

\section{B}

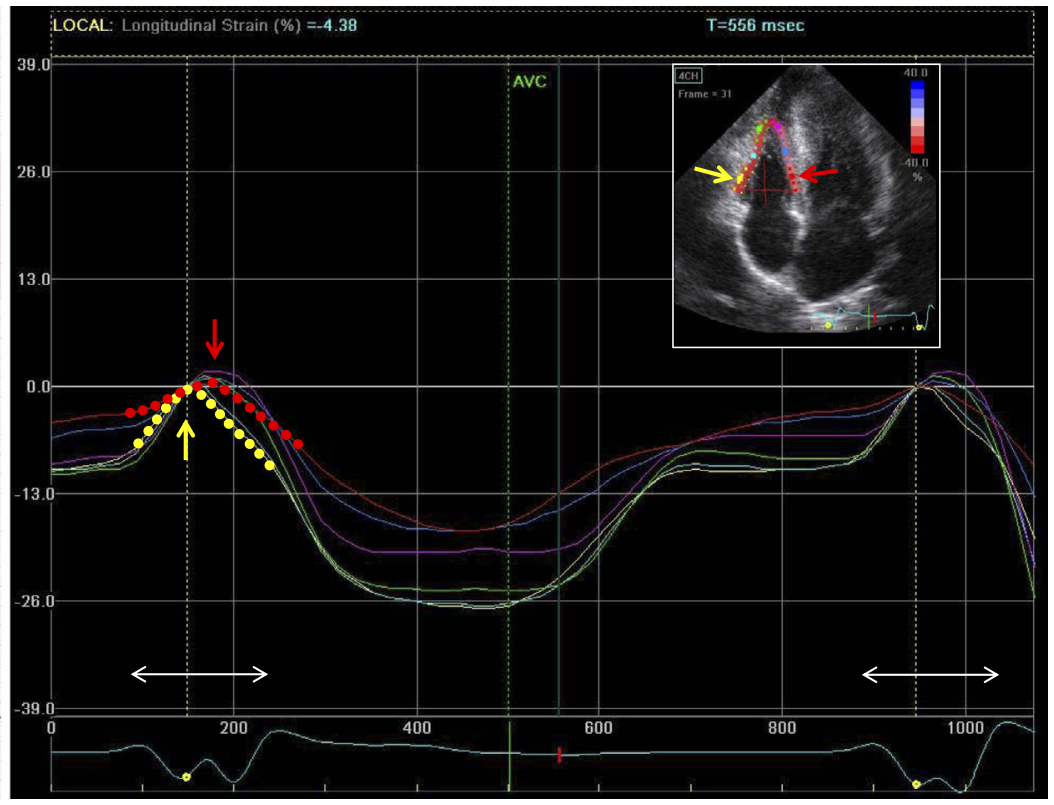

Figure 1. Twelve-lead ECG and the longitudinal strain curve in four-chamber view before catheter ablation. A: Twelve-lead ECG before catheter ablation. B: Two-dimensional speckle tracking imaging before catheter ablation. The speckle-tracking strain was obtained from a four-chamber view. The yellow arrow indicates the RV anterior wall, and the red arrow indicates the RV septal wall. The peak systolic longitudinal strain of the RV anterior wall was earlier than that of the septal wall. The ECG showed a wide QRS interval (white arrows).
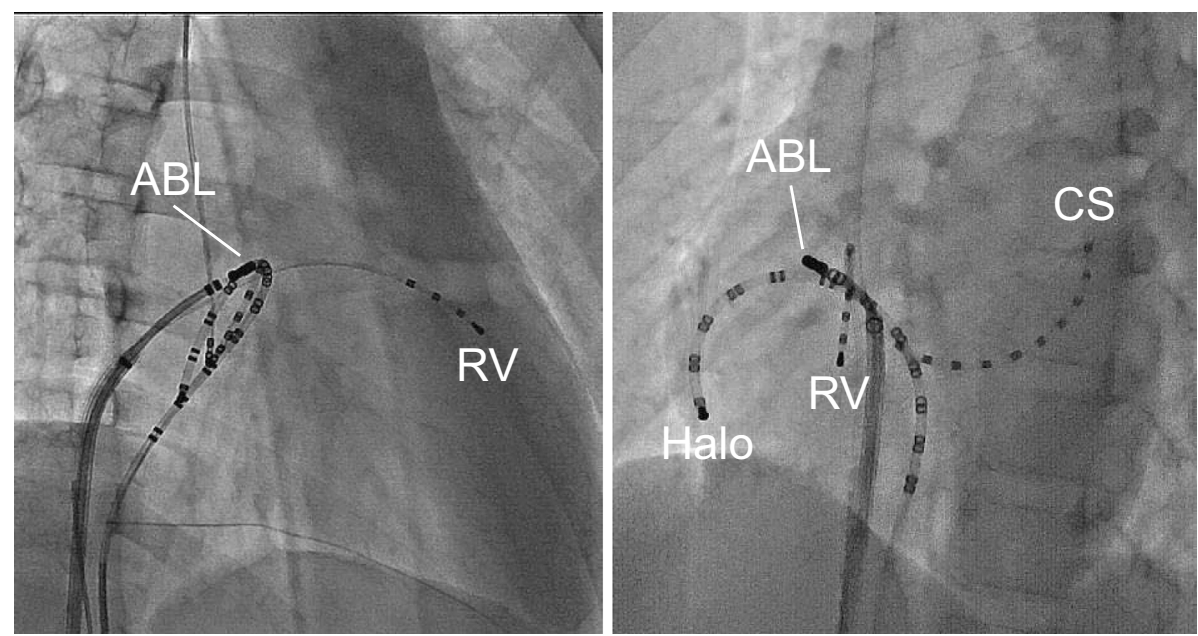

Figure 2. Successful ablation site. Radiographs obtained in the right anterior oblique (RAO, $35^{\circ}$; left panel) and left anterior oblique ( $\mathrm{LAO}, 4^{\circ}$; right panel) projections showing the ablation catheter (ABL), coronary sinus catheter (CS), Halo catheter (Halo), and right ventricular catheter (RV). ABL indicates the successful ablation site.

has been observed during a two-year follow-up period.

\section{Discussion}

Several ECG algorithms have been established and are available for patients with WPW syndrome who do not have structural heart disease. However, the accuracy of identifying accessory pathway locations by 12 -lead ECG is limited in patients with heart disease, including ventricular hypertro- phy $(1,2)$. A non-invasive strategy using M-mode echocardiography (3) and tissue Doppler echocardiography (4) has been used to localize accessory pathways in patients with WPW syndrome. Tissue Doppler echocardiography has high diagnostic accuracy in patients with left-side, but not rightside accessory pathways (4). Although we investigated this patient using these non-invasive strategies, they did not accurately localize the accessory pathway. Recently, twodimensional speckle tracking imaging has been used to diag- 
A

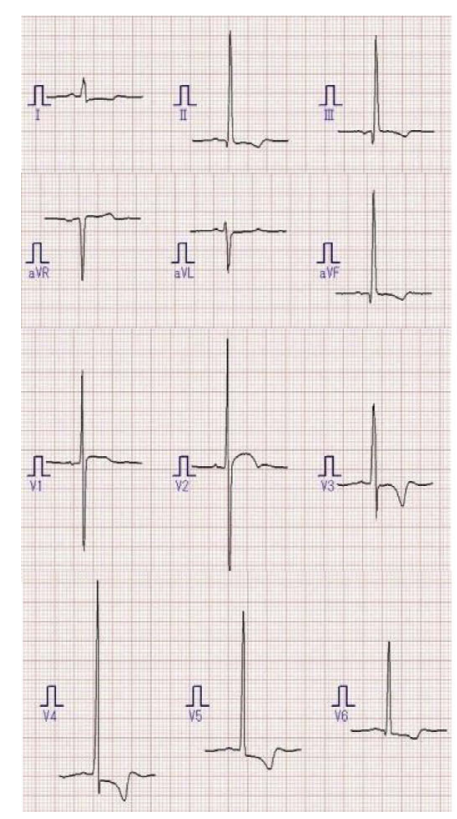

B

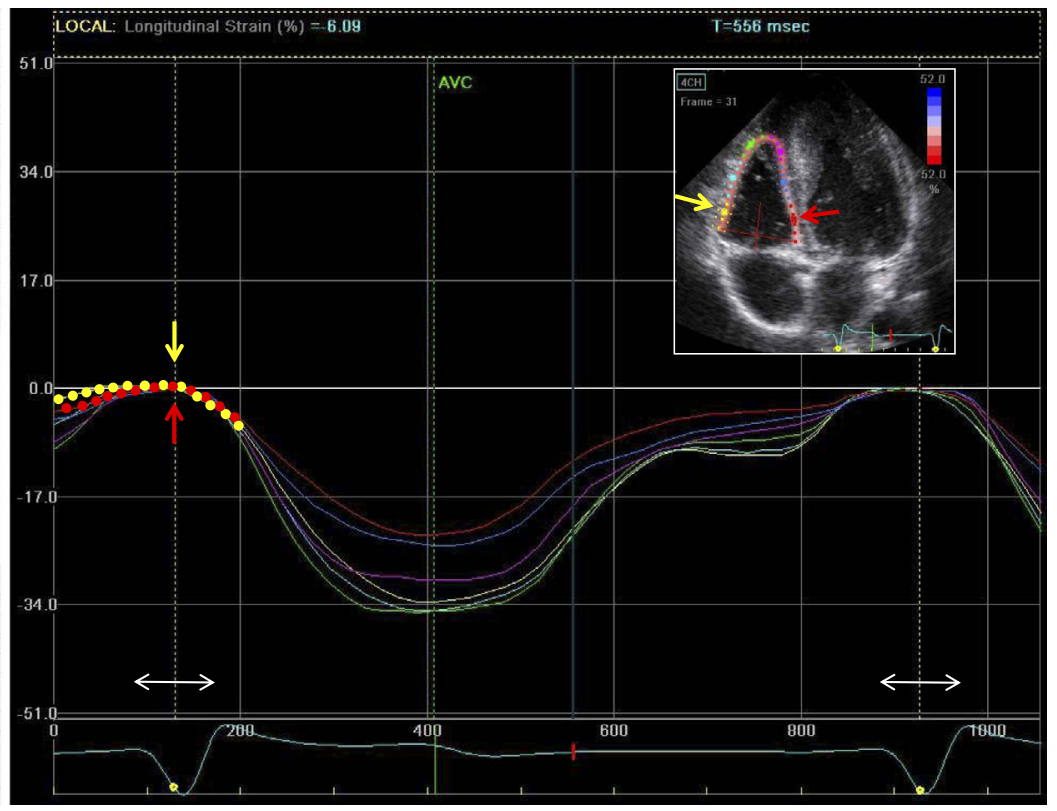

Figure 3. Twelve-lead ECG and the longitudinal strain curve in the four-chamber view after catheter ablation. A. Twelve-lead ECG after catheter ablation. B. Two-dimensional speckle tracking imaging after catheter ablation. The speckle-tracking strain was obtained from a four-chamber view. The yellow arrow indicates the RV anterior wall, and the red arrow indicates the RV septal wall. After radiofrequency catheter ablation, the $R V$ longitudinal strain showed normalization of the $R V$ regional deformation and narrowing of the QRS interval (white arrows).

nose myocardial deformation and dyssynchrony in some clinical settings $(5,6)$. It has the advantage of angle independence and may be superior to tissue Doppler-based estimation. In addition, two-dimensional speckle tracking imaging can be used to evaluate RV regional deformation (7). De Boeck et al. first reported the usefulness of the twodimensional speckle tracking imaging technique to estimate the origin of a left-side accessory pathway in a 51-year-old man (8). Recently, Delelis et al. reported that twodimensional speckle tracking findings accurately matched the endocardial mapping findings for localizing accessory pathways ( $15 \%$ were on the right side) (9). However, these study subjects did not have structural heart diseases. In the present case, we assessed a patient with significant LV hypertrophy and a right-side accessory pathway. Twodimensional speckle tracking imaging provided helpful information to identify the location of the accessory pathway before we performed catheter ablation. The RV radial and transverse strain could not demonstrate ventricular wall deformation because the RV ventricular wall was too thin. We could speculate the location of the accessory pathway, which could shorten the procedure times; however, the precise location of the earliest RV motion could not be identified.

Two-dimensional speckle tracking imaging may therefore be a useful strategy to shorten the fluoroscopy and procedure times of catheter ablation in a patient with an accessory pathway, even those who have significant LV hypertrophy and a right-side pathway.
The authors state that they have no Conflict of Interest (COI).

\section{References}

1. Basiouny T, de Chillou C, Aliot E. Accuracy and limitations of published algorithms using the twelve-lead electrocardiogram to localize overt atrioventricular accessory pathways. J Cardiovasc Electrophysiol 10: 1340-1349, 1999.

2. Takahashi N, Shinohara T, Hara M, Saikawa T. Wolff-ParkinsonWhite syndrome concomitant with idiopathic ventricular fibrillation associated with inferior early repolarization. Intern Med 51: 1861-1864, 2012.

3. DeMaria AN, Vera Z, Neumann A, Mason DT. Alterations in ventricular contraction pattern in the Wolff-Parkinson-White syndrome. Detection by echocardiography. Circulation 53: 249-257, 1976.

4. Eder V, Marchal C, Tranquart F, Sirinelli A, Pottier JM, Cosnay P. Localization of the ventricular preexcitation site in WolffParkinson-White syndrome with Doppler tissue imaging. J Am Soc Echocardiogr 13: 995-1001, 2000.

5. Kanzaki H. Mechanical dyssynchrony is not everything of substrate but is essential for cardiac resynchronization therapy. Is assessment of mechanical dyssynchrony necessary in determining CRT indication? (Pro). Circ J 75: 457-464, 2011.

6. Ishizu T, Seo Y, Baba M, et al. Impaired subendocardial wall thickening and post-systolic shortening are signs of critical myocardial ischemia in patients with flow-limiting coronary stenosis. Circ J 75: 1934-1941, 2011.

7. Tugcu A, Yildirimtürk O, Tayyareci Y, Demiroglu C, Aytekin S. Evaluation of subclinical right ventricular dysfunction in obstructive sleep apnea patients using velocity vector imaging. Circ J 74: 312-319, 2010.

8. De Boeck BW, Cramer MJ, Loh P, Doevendans PA. Images in 
Intern Med 53: 1527-1530, 2014 DOI: 10.2169/internalmedicine.53.2019

cardiovascular medicine. Two-dimensional strain imaging to assess the origin and extent of ventricular preexcitation associated with an accessory bypass. Circulation 113: e835-e839, 2006.

9. Delelis F, Lacroix D, Richardson M, et al. Two-dimensional speckle-tracking echocardiography for atrioventricular accessory pathways persistent ventricular pre-excitation despite successful radiofrequency ablation. Eur Heart J Cardiovasc Imaging 13: 840$848,2012$.

(C) 2014 The Japanese Society of Internal Medicine http://www.naika.or.jp/imonline/index.html 